\title{
AGN dusty plasma polarization features
}

\author{
Ericson D. Lopez ${ }^{1,2}$ and Susana Deustua ${ }^{2}$ \\ ${ }^{1}$ Observatorio Astronómico de Quito/Escuela Politécnica Nacional, \\ Interior del Parque La Alameda, Av. Gran Colombia s/n, 17-01-165, Quito, Ecuador \\ email: ericsson.lopez@epn.edu.ec \\ ${ }^{2}$ Space Telescope Science Institute \\ 3700 San Martin Dr., Baltimore, MD 21218, USA \\ email: deustua@stsci.edu
}

\begin{abstract}
We present the results of a theoretical study on the influence of dust particles on the polarization properties of the radiation that propagates along the jet in AGNs. First, a model for describing the interaction of dust particles, in addition to the electrons and ions, with electromagnetic radiation in a magneto-active plasma has been developed. From here, the contribution of dust particles to the Faraday rotation of the plane of polarization of the electric vector can be deduced. This model is evaluated for the outer region of the jet where the presence of dust particles are assumed, the magnetic field is weak and the electron density is low. Our results show that the dust particles give a significant contribution to the linear Faraday rotation measure.
\end{abstract}

Keywords. galaxies: active, dust, polarization

\section{Introduction}

Faraday rotation provides a direct diagnostic for the presence of magnetized material through observations of the change in the state of polarization with frequency as the radiation passes through the magneto-ionic media along the line of sight. The normal rotation of the plane of polarization as it propagates through a region with free electrons and a magnetic field is expected to be affected by the presence of ions and dust particles, which are also components of the Faraday material. Although, in general, the contribution of dust particles to the Faraday polarization effect is less significant, there are situations where they could affect the polarization properties of the radiation, in competition with the electrons.

Elongated charged dust grains are ubiquitous in cosmic plasmas (Spitzer 1997; Harwit 1988). Dust grains can acquire rotational and spinning motions due to their interaction with photons and particles in the surrounding gas or due to the presence of an oscillating electric field in a plasma (Tskhakaya \& Shukla 2001). In astrophysical objects the angular frequency of the dust grain rotation can reach a rather large value, between tens of $\mathrm{kHz}$ to $\mathrm{MHz}$ for thermal dust grains and hundreds and thousands of $\mathrm{MHz}$ for super thermal grains (Spitzer 1997, Harwit1988).

Direct emission from dust can be detected at FIR and sub-mm wavelengths where the thermal re-radiation from dust grains is expected for quasars and radio galaxies. Polarization observations are necessary to estimate the amount of dust material present in the Faraday medium and determining its contribution to the angular rotation of the electric polarization vector.

In this current contribution, we investigate the influence of dust particles in the quasar jet plasma on the polarization features of the radiation which is propagating along the astrophysical outflow. Part one presents the dispersion relations which describe the be- 
havior of the electromagnetic radiation propagating in dusty plasma. The second part is dedicated to investigating the Faraday rotation effect due to the contribution of the dusty particles that is supposed to exist in the outer kilo-parsec regions of the quasar jets.

\section{Dust properties}

A plasma with dust particles or grains can be termed as either dust in a plasma or a dusty plasma depending on the ordering of a number of characteristic lengths (Verheest 2000, Shukla \& Mamun 2002). When the inter-grain distance is greater than the grain radius and less than the Debye characteristic radius, i.e., a system where the charged dust particles participate in the collective behavior of the plasma and its electromagnetic radiation, we call this a dusty plasma. At low temperatures, a dusty plasma is fully or partially ionized, and its constituents are electrons, ions, charged dust grains and neutral atoms. Dust grains are massive (billions times heavier than the protons) and their sizes range from nanometers to millimeters (and may be as large as a micron). The dust grains in interstellar or circumstellar clouds are dielectric ices, silicates, and metallic graphite, magnetite, amorphous, carbons, etc. Particles of amorphous carbon and amorphous silicates are most commonly considered as the major source of the infrared radiation observed from carbon and oxygen stars (Gail \& Sedlmayr 2004, Jager et al. 1994). They are not neutral but are charged either negatively or positively depending on their surrounding plasma environment. Typically, a dust grain acquires between one thousand to several hundred thousand of elementary charges.

In a dusty plasma, the interaction between dust grains is screened by the background electrons and ions. The electrons oscillate around the ions at a typical electron plasma frequency $w_{p e}$. The ions oscillate around charged dust grains at the ion plasma frequency $w_{p i}$ and the dust particles oscillate around their equilibrium position with a dust plasma frequency $w_{p d}$. Typically, due the large mass and charge of the dust particles, the dust plasma or cyclotron frequencies are generally many orders of magnitude smaller than corresponding ion frequencies and those in turn are smaller than the electron plasma frequencies. Consequently, the dust frequencies are often considered negligible in electromagnetic calculations.

\section{Faraday effect for a dusty plasma}

In this section, we delineate the procedure to obtain the expression for the Faraday rotation effect in a dusty, magneto-active plasma. The detailed analysis of the oscillations of a collisionless magneto-active plasma is very complex since the number of solution branches is practically infinitely large compared to the number of electromagnetic modes existing in an unmagnetized plasma. This complexity is even larger when dust particles are included. The presence of charged particles is found to modify or even dominate wave propagation in multicomponent dusty plasma where the collective processes are of great importance. The presence of charged dust grains does not only modify the existence of low-frequency waves (e.g., ion-acoustic waves), but also introduces new types of dustrelated waves (Shukla \& Mamun 2002).

In the current contribution we consider the cold approximation, when the thermal motion of the particles is entirely neglected. We follow the kinetic theory that provides a detailed microscopic description of plasma. The theory is able to treat with great accuracy the behavior of the constituent particles, their interactions and the influence of external electromagnetic fields. The response of a dusty plasma to the electromagnetic 
perturbation is mainly characterized by the dielectric permittivity parameter, which for an anisotropic plasma must be a tensor. Consequently, in our first approximation, we determine the explicit form for the dielectric tensor of permittivity corresponding to a homogeneous collisionless magneto-active dusty plasma. This tensor will define the kinds of electromagnetic modes which can exist and propagate in a dusty environment. Moreover, we take into account the presence of an external magnetic field which will determine the anisotropic character of the plasma and the polarization properties of the radiation propagating in the dusty medium.

We start from the general expression for the dielectric tensor of permittivity for a multicomponent plasma, given by Alexandrov et al. (1984). From this general expression it is possible to derive the corresponding relation for the dielectric permittivity tensor in a cold collision-less magneto-active dusty plasma. From the Alexandrov expression we find that the dielectric tensor is Hermitian, in agreement with the cold approximation, since the dissipative processes have been neglected. The matrix form of this permittivity tensor for dusty plasma takes the form:

$$
\epsilon_{i j}=\left(\begin{array}{lll}
\epsilon_{\perp} & i g & 0 \\
-i g & \epsilon_{\perp} & 0 \\
0 & 0 & \epsilon_{\|}
\end{array}\right)
$$

where:

$$
\begin{array}{ll}
\epsilon_{\perp}=\epsilon_{x x}=\epsilon_{y y}=1-\sum_{\alpha} \frac{w_{p \alpha}^{2}}{\left(w^{2}-\Omega_{\alpha}^{2}\right)}, & \epsilon_{\|}=\epsilon_{z z}=1-\sum_{\alpha} \frac{w_{p \alpha}^{2}}{w^{2}} \\
\epsilon_{x y}=-\epsilon_{y x}=i g=-\sum_{\alpha} \frac{w_{p \alpha}^{2} \Omega_{\alpha}}{w\left(w^{2}-\Omega_{\alpha}^{2}\right)}, & \epsilon_{x z}=\epsilon_{z x}=0 \text { and } \epsilon_{y z}=\epsilon_{z y}=0 .
\end{array}
$$

Here, $\Omega_{\alpha}, w_{p \alpha}, w$ are the resonance (cyclotron), plasma and radiation frequencies, respectively. The summation runs over each plasma component (electrons, ions and dust particles). The above expressions hold for nondegenerate and degenerate plasmas when the thermal motion of the particles is neglected. Now, the tensor $\epsilon_{i j}$ given by Eq. (1) should be replaced into the next wave equation, derived from the Maxwell equations of the electrodynamics: $\left[k^{2} \delta_{i j}-k_{i} k_{j}-\frac{w^{2}}{c^{2}} \epsilon_{i j}(w, \vec{k})\right] E_{j}=0$, where $k_{i}$ is the i-component of the wave vector $\vec{k}, E_{j}$ is the j-component of the electric vector $\vec{E}$, and $c$ is the speed of light. From here, the dispersion equation for the electromagnetic waves propagating in cold magneto-active dusty plasma is obtained by applying the determinant condition for the existence of non-trivial solutions of this physical system. This appears as a fourth order polynomial expression in $k$ :

$$
A k^{4}+B \frac{w^{2}}{c^{2}} k^{2}+C \frac{w^{4}}{c^{4}}=0
$$

where using the tensor expression (1) the coefficients $A, B, C$ are explicitly written as:

$$
A=\frac{k_{\perp}^{2}}{k^{2}} \epsilon_{\perp}+\frac{k_{z}^{2}}{k^{2}} \epsilon_{\|}, \quad B=-\frac{k_{z}^{2}}{k^{2}} \epsilon_{\|} \epsilon_{\perp}-\frac{k_{\perp}^{2}}{k^{2}}\left(\epsilon_{\perp}^{2}-g^{2}\right), \quad C=\epsilon_{\|}\left(\epsilon_{\perp}^{2}-g^{2}\right),
$$

that leads to the electromagnetic dispersion equation:

$$
n^{4}\left(\sin ^{2} \theta \epsilon_{\perp}+\cos ^{2} \theta \epsilon_{\|}\right)-n^{2}\left[\cos ^{2} \theta \epsilon_{\perp} \epsilon_{\|}+\sin ^{2} \theta\left(\epsilon_{\perp}^{2}-g^{2}\right)\right]+\epsilon_{\|}\left(\epsilon_{\perp}^{2}-g^{2}\right)=0
$$


where, $n=\left(\frac{k c}{w}\right)$ is the refraction index of the dusty medium and $\theta$ is the angle between the direction of the wave propagation and the magnetic field $\vec{B}$.

This dispersion relation (3) should be solved to obtain the diverse electromagnetic perturbations which are able to propagate in a dusty environment. For the longitudinal propagation of the electromagnetic waves (i.e., along the magnetic field, $\theta=0$ ), we obtain right and left handed circularly polarized waves propagating in cold magneto-active dusty plasma. These waves are characterized respectively by their refraction index: $n_{+}$for the ordinary wave (right polarization) and $n_{-}$for the extraordinary one (left polarization), and they are explicitly given by: $n_{ \pm}^{2}=\frac{1}{2}\left(\epsilon_{\perp} \pm \sqrt{4 g^{2}-3 \epsilon_{\perp}^{2}}\right)$.

In this result, we note that the parallel component of the dielectric tensor of permittivity is not present in these transverse waves. The parallel component is important only for describing the longitudinal modes.

On the other hand, the phase difference between the ordinary and extraordinary modes of the waves leads to rotation of the electric vector plane of polarization, when the radiation is passing through a medium with a magnetic field (Faraday Effect). Using the above results, for dusty magneto-active plasma in the cold approximation, we derive an expression for the electric vector polarization angle (EVPA) $\Psi$, which is greatly simplified considering that: $k_{\perp} V_{T_{s}} \ll w_{c s}, k_{\|} V_{T_{s}} \ll w$, and $k_{\|} V_{T_{s}} \ll\left|w \pm n w_{c s}\right|$ (i.e., in the regime where the radiation frequency $w$ is higher than the cyclotron frequency of the particles and it is also higher than the frequency related to the thermal motion of the different species). Then, with these considerations for a relatively high frequency regime, we have:

$$
\Psi=\int \frac{w^{2}}{4 c}\left(\frac{8 \Omega_{0} \Omega_{r}^{2}}{\left(\Omega_{0}-w\right)^{2}\left(\Omega_{0}+w\right)^{2}}\right)+\frac{1}{2 c}\left(\frac{w_{c e} w_{p e}^{2}}{\left(w_{c e}^{2}-w^{2}\right)}+\frac{w_{c i} w_{p i}^{2}}{\left(w_{c i}^{2}-w^{2}\right)}+\frac{w_{c d} w_{p d}^{2}}{\left(w_{c d}^{2}-w^{2}\right)}\right) d L
$$

Here, $\Omega_{0}$ is the dust rotation frequency, $\Omega_{r}=\sqrt{\frac{4 \pi r^{2} n_{d}}{4 I}}, I$ is the principal moment of inertia, $r$ is dust particle radius, $n_{d}$ is the dust concentration and $L$ is the path length that the radiation is passing through.

This is the expression that we need in order to study the dust influence on the polarization state of the electromagnetic radiation propagating in a magnetized medium where the Faraday effect is taking place. In the next section, we evaluate this EVPA relation in the astrophysical context of AGN jets.

\section{Application of the model}

In this section we evaluate expression (4) for the electric vector position angle $\psi$ as a function of frequency $w$, using the characteristic parameters for the electrons, ions and dust grains; plasma components in AGN jets. We assume that the dust particles are prolate spheroids and have an angular rotation around the $z$ axis. The magnetic field $B$ is considered to be along the jet as often seen in quasars and in particular in blazars (e.g., Pier \& Krolik 1992).

For the external regions of an AGN jet we see that $w_{c d} \ll w_{c i} \ll w_{c e} \ll w, w-\Omega_{0} \simeq w$ and $w+\Omega_{0} \simeq w$ are satisfied. Then the EVPA expression given by the Eq (4), could be approximated by:

$$
E V P A=\left[\frac{2 \Omega_{0} \Omega_{r}^{2}}{4 c w^{2}}+\frac{1}{2 c w^{2}}\left(w_{p e}^{2} w_{c e}+w_{p i}^{2} w_{c i}+w_{p d}^{2} w_{c d}\right)\right] * L\left(3.085 \times 10^{18}\right),
$$

which as a function of the wavelength $(\lambda)$ reproduces the form of the well-known linear 


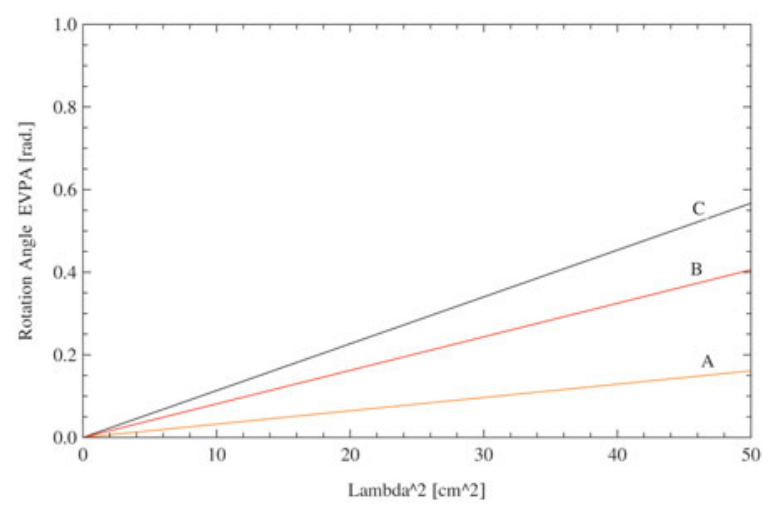

Figure 1. Faraday rotation for the kilo-parsec region of AGN jets. The red line corresponds to the RM contribution of the dust grain rotation (label B). The yellow one is the electron RM (label A) and the black line is the total Rotation Measure (label C).

expression for the Faraday lambda square law $\left(E V P A=R M \lambda^{2}\right)$. Where, the rotation measure $R M$ for the dusty plasma comes as result of the contribution by the electrons, ions, dust particles and the rotation of the dust grains. This means:

$$
R M=\left[R M_{r}+R M_{e}+R M_{i}+R M_{d}\right]
$$

with:

$$
\begin{array}{ll}
R M_{r}=5.46 \times 10^{-4} * \frac{\Omega_{0} d^{2} n_{d} L}{I}, & R M_{e}=6.736 \times 10^{-52} * \frac{B n_{e} L}{m_{e}^{2}}, \\
R M_{i}=6.736 \times 10^{-52} * \frac{Z^{3} B n_{i} L}{m_{i}^{2}}, & R M_{d}=6.736 \times 10^{-52} * \frac{Z_{d}^{3} B n_{d} L}{m_{d}^{2}} .
\end{array}
$$

For the external lobes in AGN jets we assume for the characteristic parameters of the plasma components the values given in Table 1 . the rotation frequency of the dust grains has been assumed to be about $4 \mathrm{kHz}$. The magnetic field for the external regions is considered to be around $0.5 \mu G$ and the characteristic path length $L$ of the plasma region is $200 \mathrm{kpc}$ (O'Sullivan et al. 2013). These values are based on average parameters for the interstellar dust (Verheest 2000, Evans 1994, Evans 1996).

Table 1. Mean values for characteristic parameters of dusty plasma in the external regions of AGN jets

\begin{tabular}{|l|l|l|l|}
\hline Parameters & Electrons & Ions & Dust \\
\hline Mass density $\left(\mathrm{g} \mathrm{cm}^{-3}\right)$ & & & 2.2 \\
Number density $\left(\mathrm{cm}^{-3}\right)$ & $1 \times 10^{-4}$ & $4 \times 10^{-4}$ & $1 \times 10^{-7}$ \\
Charge $(\mathrm{z})$ & $e$ & $Z_{i}=1$ & $4 \times 10^{4}$ \\
Mass $(\mathrm{g})$ & $m_{e}$ & $m_{H}$ & $1 \times 10^{-12}$ \\
Temperature $(K)$ & & $r_{H}$ & 300 \\
Radius $(\mu \mathrm{m})$ & $r_{e}$ & $r_{H}$ & 0.2 \\
\hline
\end{tabular}

Figure (1) shows the EVPA vs. $\lambda^{2}$ for the kilo-parsec region of the AGN Jets. We see that the contribution of the dust grain rotation to the Faraday effect is significantly higher than the electron component. The total EVPA is the sum of the RMs of each component of the dusty plasma. The ion gyro-frequency is about $4.8 \times 10^{-3} \mathrm{~Hz}$, the dust grain gyro-frequency is about $3.2 \times 10^{-10} \mathrm{~Hz}$, values which are much less than the electron gyro-frequency of $\sim 8.8 \mathrm{~Hz}$. 


\section{Discussion and Conclusions}

The precise study of plasma properties, in general, is a very complex problem due to the microscopic description of the large numbers of electrons and ions that interact with each other, with the surrounding electromagnetic radiation and external magnetic fields. This situation is further complicated when considering additionally the presence of dust particles, as these systems are still poorly understood and require many more theoretical and experimental studies to gain a better understanding of the nature of the radiation exists and propagates through a dusty medium. To date, relatively few studies have been made to understand the nature of dusty plasma. In this paper we have tried to clarify the effect dust particles exert on dispersive effects such as on the rotation measure.

In this context, after developing a kinematic model, an expression for the angle of rotation of the polarization vector for dusty plasma has been derived. This expression is evaluated for the outer regions on kilo-parsec scales where the lobes are considered to contain significant amounts of dust. The results show, in the outer regions where the magnetic field is weaker and the electron concentration decreases greatly, the contribution of electrons still dominates over the ions and dust particles. However, when elongated dust particles in a magnetic field have rapid rotation $(\mathrm{kHz}$ and hundreds and thousands of $\mathrm{MHz}$ ) the Faraday rotation measurement is affected by the rotation of dust particles, which can be a larger effect on polarization than that produced by the electrons. In conclusion, Faraday rotation is not affected by dust particles alone, but can be due to the rapid rotation that these dust particles may acquire during the electric charging processes.

Since polarization observations at millimeter wavelengths are most promising and can provide more accurate information about the polarization regime in these systems, where the large rotation measure values are expected, coming ALMA observations at $\mathrm{mm}$ wavelengths will be of great importance to study the dust in the torus and along the jet and its influence on the radiation propagating through the dusty magnetized plasma.

\section{References}

Alexandrov, A. F., Bogdankevich, L. S., \& Rukhadze, A. A. 1984, Principles of Plasma Electrodynamics (Berlin: Springer)

Evans, A. 1994, The Dusty Universe (Chichester: Wiley)

Evans, A. 1996, SSpace Sci. Revs, 75, 620

Gail, H. P. \& Sedlmayr, E. 2004, A\&A, 133, 320

Harwit, M. 1988, Astrophysical Concepts (New York: Springer)

Jager, C. et al. 1994, A\&BA, 292, 641

O' Sullivan, S. P, Feain, I. J., McClure-Griffiths, N. M., et al. 2013, ApJ, 764, 162

Pier, E. A. \& Krolik, J. H. 1992, ApJ, 399, L23

Shukla, P. K. \& Mamun, A. A. 2002, Introduction to Dusty Plasma Physics (London: IOP)

Spitzer, L. Jr. 1997, Physical Processes in the Interstellar Medium (New York: Wiley)

Tskhakaya, D. \& Shukla, P. K. 2001, Phys. Lett. A, 279, 456

Verheest, F. 2000, Waves in Dusty Space Plasma (Netherlands: Kluwer) 